Musées, Patrimoine et Culture scientifiques et techniques

$162 \mid 2015$

novembre-décembre 2015

\title{
Numériser pour préserver et valoriser les patrimoines culturels
}

\author{
Jean-Louis Blanchart
}

\section{OpenEdition \\ Journals}

Édition électronique

URL : http://journals.openedition.org/ocim/1581

DOI : 10.4000/ocim. 1581

ISSN : 2108-646X

\section{Éditeur}

OCIM

Édition imprimée

Date de publication : 1 novembre 2015

Pagination : 20-24

ISSN : 0994-1908

Référence électronique

Jean-Louis Blanchart, « Numériser pour préserver et valoriser les patrimoines culturels », La Lettre de I'OCIM [En ligne], 162 | 2015, mis en ligne le 01 novembre 2016, consulté le 19 avril 2019. URL : http:// journals.openedition.org/ocim/1581; DOI : 10.4000/ocim.1581

Ce document a été généré automatiquement le 19 avril 2019

Tous droits réservés 


\title{
Numériser pour préserver et valoriser les patrimoines culturels
}

\author{
Jean-Louis Blanchart
}

La page d'accueil du portail numeriques.be

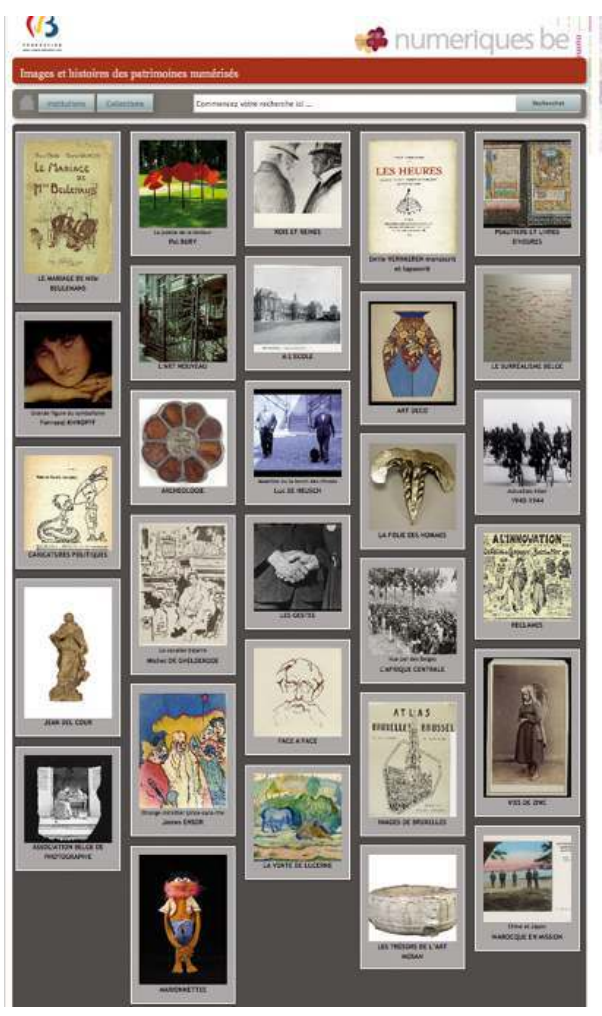

La numérisation a un impact important sur les modes de production et de diffusion des œuvres et objets de tous les secteurs culturels. Elle modifie aussi la manière dont le public aborde ces œuvres et objets. La combinaison, favorisée par la numérisation, des trois « dé » - pour « dématérialisation, déchronologisation et désintermédiation » - a ouvert la voie aux investissements d'acteurs jusque-là peu ou pas actifs dans les secteurs culturels, 
tels que les opérateurs de réseau, des équipementiers ou des acteurs dits " over the top " (moteurs de recherche, plateforme de vidéo en ligne, réseaux sociaux).

2 Les institutions culturelles, dont les musées et les centres d'archives, n'ont que récemment pris la mesure des effets de cette mutation technologique sur la consommation des biens culturels par le public, poussés dans le dos par les initiatives d'acteurs comme Google, qui a réussi à imposer son moteur de recherche ainsi que son système de référencement, et qui a besoin de contenus culturels (Google Art par exemple) pour développer son modèle économique.

3 Des études le montrent, de gros progrès restent à faire dans le domaine de la numérisation du patrimoine culturel $^{1}$. Ce retard pris dans la numérisation des patrimoines culturels est sans doute l'une des raisons principales de l'impact encore peu déterminant - en tout cas pour ce qui concerne la Fédération Wallonie-Bruxelles - de la numérisation sur la gestion des musées et des centres d'archives.

4 En tout état de cause, il appartient aux pouvoirs publics d'investir dans la numérisation des patrimoines culturels, et ce pour plusieurs raisons :

5 - la numérisation des patrimoines culturels nécessite des moyens financiers importants, et ce pour une espérance de rentabilité fort aléatoire ;

6 - dans le cas où une rentabilité est attendue, une numérisation sur fonds privés peut faire courir le risque d'une privatisation de l'accès à des collections qui, souvent dans le milieu des musées, sont dans le domaine public.

7 Pour ce qui concerne la Fédération Wallonie-Bruxelles (FW-B), la nécessité de donner un cadre stratégique et financier à l'action des pouvoirs publics a été reconnue dans le contexte d'un plan adopté en 2007: il s'agit du plan PEP's (pour Préservation et Exploitation des Patrimoines).

\section{Le plan PEP's}

8 Le plan PEP's a été adopté par le Gouvernement de la FW-B en octobre 2007 (PEP's 2007). Il s'agit de l'acte fondateur de la politique menée par la FW-B en matière de numérisation de son patrimoine culturel, ainsi que de préservation et de valorisation des objets numérisés. Il a l'ambition de regrouper l'ensemble des institutions de la FW-B, généralement de petite taille, et dont les moyens pour numériser et gérer la préservation du patrimoine et des collections parfois très éparpillées ne sont pas toujours suffisants. L'idée d'une mutualisation des moyens et d'une organisation de la numérisation autour de standards communs s'est dès lors imposée afin de préserver cette richesse.

9 Le plan PEP's est ainsi un plan de numérisation des collections et fonds culturels et patrimoniaux conservés dans les musées, les centres d'archives, les bibliothèques, les institutions audiovisuelles. Il a pour double objectif :

10 - la préservation des patrimoines culturels en veillant à la sauvegarde et à la pérennité des collections afin de continuer à avoir accès à court et moyen termes aux patrimoines numérisés ;

11 - la valorisation des patrimoines culturels en assurant un accès interopérable via un portail pour les services et institutions de la Communauté française, le grand public, les réseaux d'enseignement et les chercheurs. 
Les entrées par institution et par collection.
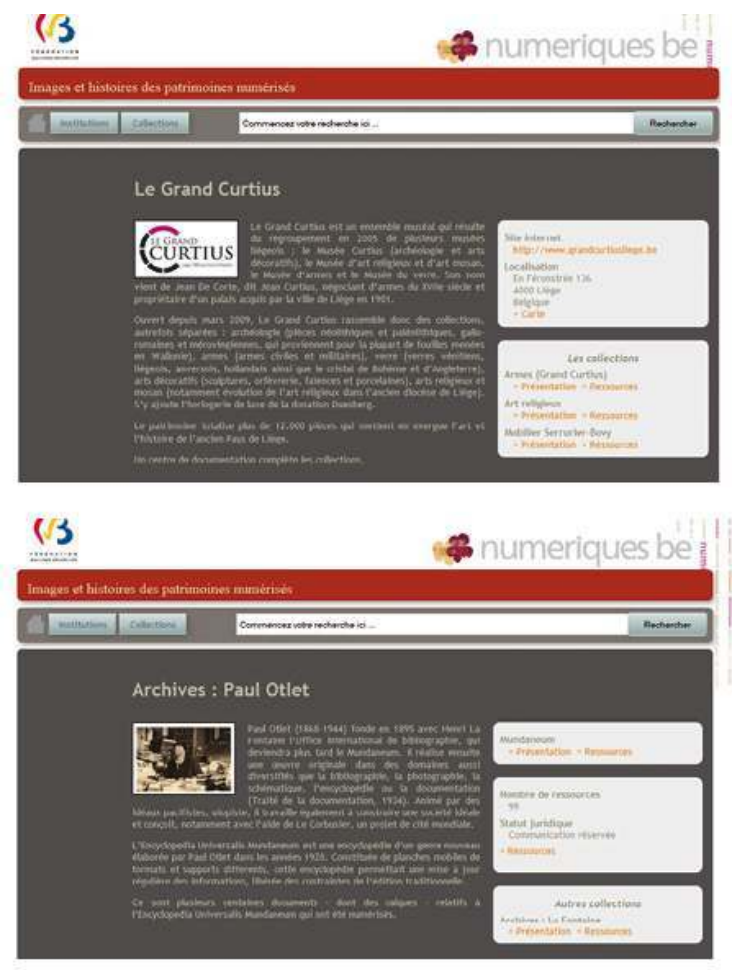

$$
13 \text { - presen }
$$

- préservation à long terme : la numérisation doit se faire directement sous deux formats : valorisation (par exemple pdf, jpeg) et conservation (par exemple tiff, jpeg2000). Qui plus est, une infrastructure de préservation à long terme basée sur la norme OAIS ${ }^{2}$ devrait idéalement être mise en place : en effet, il ne peut être envisageable pour un pouvoir public responsable de recommencer les opérations de numérisation tous les dix ans;

14 - ouverture: le choix par les institutions des formats, modèles et applications doit favoriser l'interopérabilité des métadonnées de manière à faciliter la mise en commun de ressources et la recherche croisée sur plusieurs bases de données;

15 - accessibilité : la numérisation doit permettre de mettre en contact un patrimoine riche et diversifié avec un public large et diversifié, gratuitement pour les œuvres hors droits numérisées sur des fonds publics;

16 - droits d'auteur : la numérisation s'inscrit pleinement dans les législations en vigueur ;

17 - éditorialisation : la numérisation n’a de réel intérêt que si elle est accompagnée par des mises en valeur documentaires et éditoriales qui doivent être innovantes et se renouveler en fonction des publics concernés.

18 La délégation générale à la numérisation des patrimoines culturels, créee en 2008 dans la foulée de ce plan, est en charge de sa mise en œuvre. La délégation générale assure ainsi plusieurs missions, dont les plus importantes sont la gestion de la numérisation des patrimoines culturels, ainsi que la préservation des patrimoines numérisés et l'accès en ligne à ces mêmes patrimoines. Outre ces missions de base, la délégation générale peut aider les institutions culturelles dans la réalisation de leurs propres projets de numérisation. À cet effet, elle publie sur son site www.peps.cfwb.be un guide Normes et 
lignes directrices techniques et organisationnelles pour les initiatives de numérisation des patrimoines culturels soutenues par la Communauté française (PEP's 2009), des recommandations sur l'organisation et la structuration des métadonnées et un document de synthèse sur la gestion des droits d'auteur et droits voisins dans l'univers numérique.

Une recherche par lieu

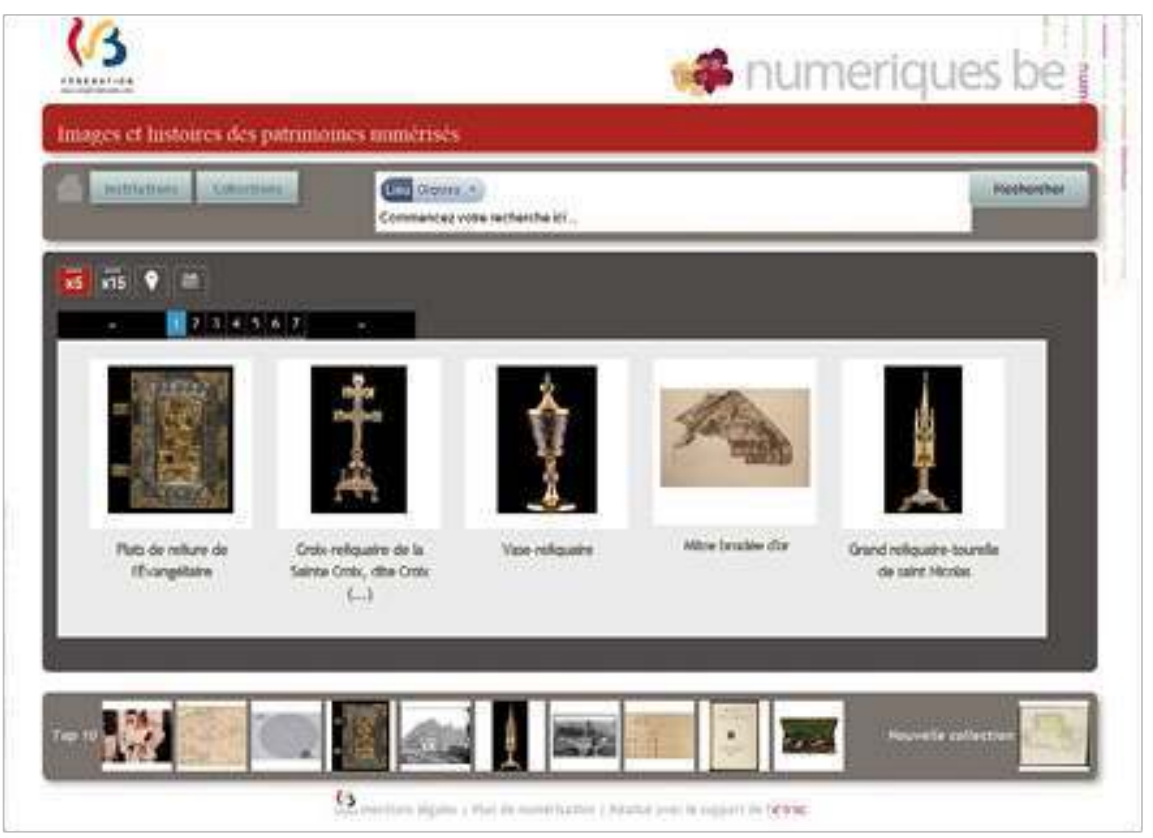

\section{La numérisation des patrimoines culturels}

\section{culturelles est réalisée par des sociétés spécialisées. Celles-ci se basent sur le fichier de} récolement qui reprend l'identifiant unique des pièces et de leur description ainsi que leur état. La délégation générale préconise que le fichier de récolement soit aussi proche que possible des métadonnées descriptives sans lesquelles le risque est grand de ne plus retrouver les œuvres numérisées et de ne pas les exploiter. Dans le cadre du Plan PEP's, toute numérisation est demandée en deux formats, un format dit de conservation, dans une très haute résolution et dans des normes destinées à être pérennes (tiff ou jpeg2000 par exemple), et un format de valorisation exploitable sur Internet (pdf/a ou jpeg par exemple).

Un point important est que les institutions doivent absolument fournir les métadonnées associées aux objets numérisés. Ces métadonnées sont effet indispensables pour l'identification des objets, leur recherche sur internet et la gestion de leur préservation à 
long terme. Dans ce contexte, la délégation offre aux institutions un support sous deux formes :

23 - dans le cas où les institutions n'ont pas de base de données pour la gestion des métadonnées, la délégation peut leur offrir un tableur Excel pré-formaté reprenant les champs de métadonnées indispensables pour une gestion de base, ainsi qu'un mode d'emploi d'encodage des métadonnées ;

24 - si les institutions ont leur propre base de données, la délégation leur demande de lui fournir une sortie de cette base de données au format XML, ce format étant ouvert universel et pérenne. Elle se charge ensuite de toutes les opérations de transcodage dans le schéma de métadonnées qu'elle utilise pour la mise en ligne sur son portail.

Ces campagnes de numérisation ont été lancées, soit sur base de demandes introduites par les institutions culturelles, soit à l'initiative de la FW-B elle-même. Pour donner un exemple de ce dernier cas de figure, en 2013-2014, la FW-B a souhaité mettre la priorité sur la numérisation des biens culturels mobiliers classés en tant que trésors de la Communauté française. Entre 2008 et 2014, un budget cumulé de près de 1,7 millions d'euros a été consacré à la numérisation de collections appartenant à 32 institutions culturelles. Ont été numérisés pour ce budget plus de 500000 pages d'origines diverses (livres, revues, archives...), plus de 65000 photographies, plus de 1400 peintures, dessins et gravures, plus de 8000 plans et affiches, 250 heures de vidéo, plus de 1000 heures de sons (musique, interviews...) et environ 12000 pièces de tout genre (archéologie, sculptures, armes...).

\section{La préservation des patrimoines numérisés}

Après un contrôle qualité réalisé par les sociétés qui numérisent et par la délégation et les institutions, les fichiers (représentations numériques des objets, et métadonnées associées) sont ensuite conservés selon les normes internationales en vigueur pour la préservation numérique à long terme, c'est-à-dire sur au moins trois supports de stockage différents entreposés en deux lieux distincts: les institutions gardent au minimum une copie des fichiers sur disque dur externe, tandis que la délégation garde deux copies : une sur disque dur externe et l'autre sur cartouches LTO $^{3}$.

Nous l'avons déjà dit, "numérique " ne veut pas dire "pérennité ", et ce pour de multiples raisons (obsolescence rapide des formats, dégradation des supports de stockage...). Il est donc nécessaire de mettre en place des stratégies basées sur la norme OAIS et visant à assurer la préservation à long terme des fichiers numérisés ; le stockage sur cartouches LTO avec contrôle régulier de l'intégrité des fichiers fait partie de ces stratégies. 
Un exemple de fiche

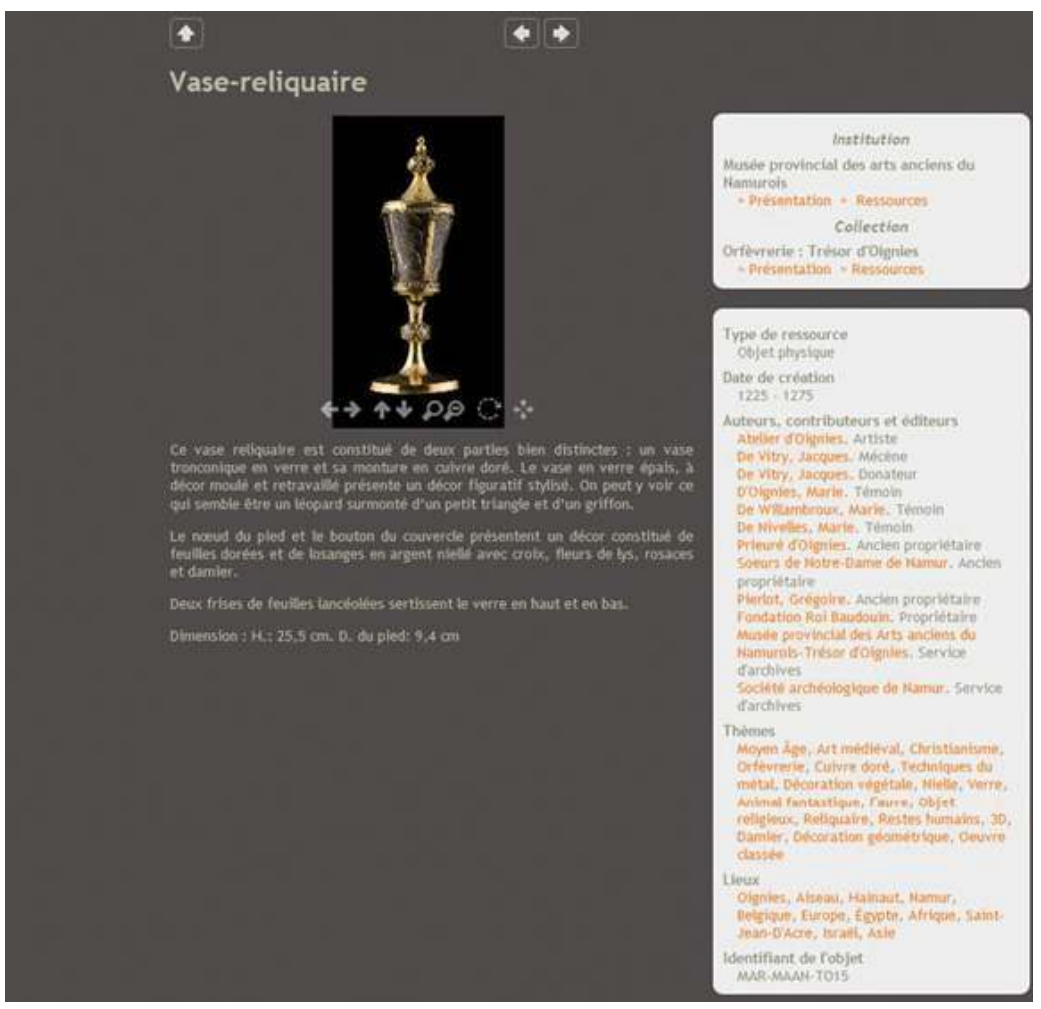

\section{L'accès aux patrimoines numérisés et le portail www.numeriques.be}

Les pouvoirs publics ont aussi dégagé des moyens pour développer un portail d'accès aux patrimoines numérisés: www.numeriques.be a été lancé officiellement en novembre 2013.

29 Le portail propose au public de découvrir les patrimoines en suivant plusieurs pistes, selon sa curiosité :

30 - l'onglet «Institutions » donne accès à une présentation de l'institution ainsi qu'à l'ensemble des ressources disponibles par collections pour cette institution. 34 institutions sont actuellement présentes dans le portail ;

31 - l'onglet «Collections » donne accès à une présentation de chaque collection ainsi qu'à l'ensemble des ressources disponibles pour cette collection. Il y a actuellement 76 collections ;

32 - un moteur de recherche donne accès aux œuvres numérisées par mots clés ou par recherche plein texte. Cet accès est largement facilité par l'interopérabilité des métadonnées organisée en amont ainsi que l'organisation des métadonnées par Personne, Lieu, Sujet, Organisation. Les œuvres correspondant à la recherche sont présentées par des vignettes, par leur localisation et dans une ligne du temps. En cliquant sur l'œuvre souhaitée, l'internaute a accès aux métadonnées, qui peut mener vers d'autres découvertes, et à une représentation de l'œuvre, parfois en 3D, parfois en zoom progressif à très haute résolution ; 
33 - au départ de la page d'accueil, en cliquant sur les vignettes, l'internaute a accès à des parcours de découverte, alliant les collections, l'image, le texte, le son et la vidéo selon des entrées par thèmes. Un lien permet de retourner à la description de l'œuvre dans le moteur de recherche.

34 Toutes ces entrées interagissent, croisent les métiers, les regards, les lieux et les époques. Pour ce faire, un long et fastidieux travail sur les métadonnées a été réalisé par l'équipe de la Délégation générale à la numérisation des patrimoines culturels, en charge de la mise en œuvre du Plan PEP's. Il s'agit d'enrichir et de rendre interopérable la manière dont chacune des institutions décrit ses collections afin d'éliminer le bruit et de rendre la recherche plus fluide et plus riche.

35 Enfin, la promotion de collections numérisées et du portail est également assurée par le biais :

36 - d'une page Facebook ouverte en avril 2014 - Numériques.be - et proposant entre autres des liens entre objets et actualités, des associations d'images... ;

37 - d'une lettre d'information lancée en mai 2015, et proposant de courtes histoires centrées sur des objets du portail.

\section{Des projets pour l'avenir}

\section{Un accès vers Europeana}

Le portail www.numeriques.be est le point de convergence des collections numérisées des institutions de la FW-B. Il sera aussi le point d'injection de ces collections sur le portail européen www.europeana.eu. La délégation se chargera des opérations techniques nécessaires pour cette exposition complémentaire.

39 Le patrimoine culturel est d'autant plus vivant que son accessibilité est démultipliée, et Europeana offre à cet égard une surface d'exposition européenne.

\section{En route vers le Web sémantique}

Le Web sémantique (ou Web de données) est le Web de demain. Son objectif est de faire en sorte que l'utilisateur puisse rechercher, partager et combiner l'information plus facilement. Il s'appuie sur un ensemble de standards et de technologies $\left(\mathrm{RDF}^{4}, \mathrm{OWL}^{5}\right)$ développé par le $\mathrm{W} 3 \mathrm{C}$ visant à structurer les données et à en faciliter l'exploitation notamment en permettant leur interprétation par des machines. 


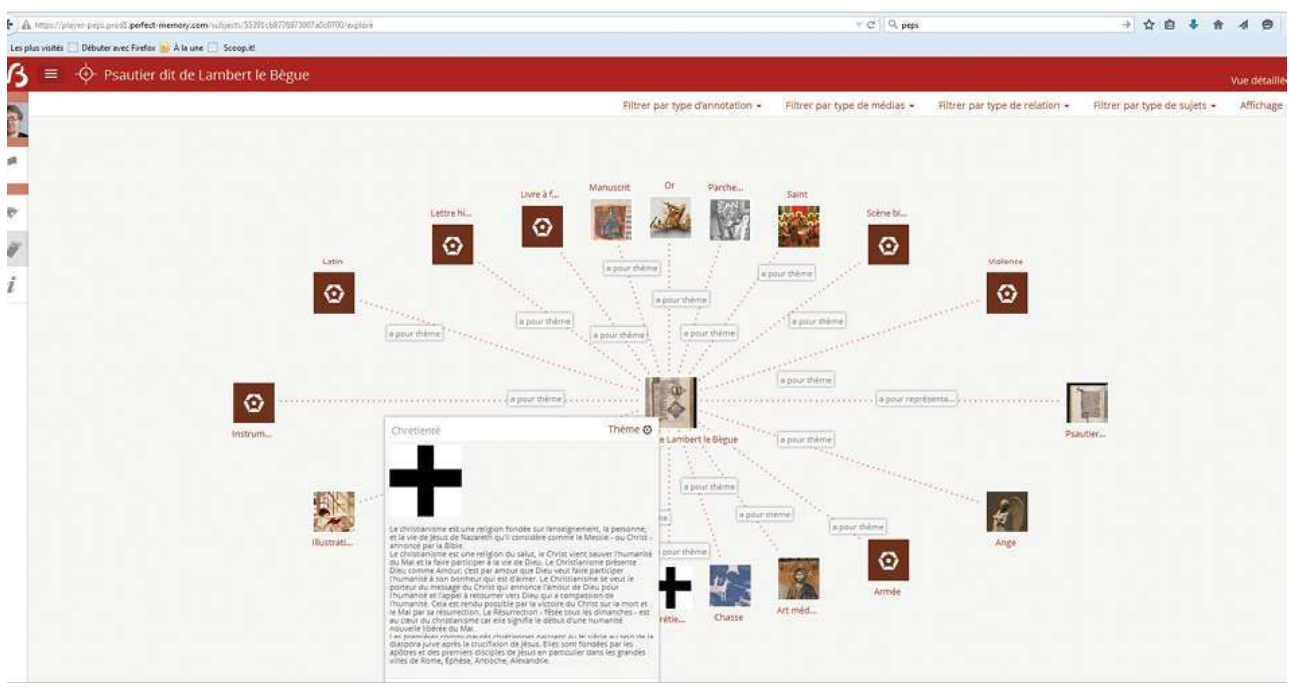

Les institutions culturelles comme les bibliothèques et les musées produisent depuis longtemps des données structurées pour leurs catalogues. Elles sont donc par nature des acteurs potentiels du Web sémantique. Des projets existent déjà dans d'autres pays (par exemple le projet Jocondelab en France). La Fédération Wallonie-Bruxelles a voulu s'inscrire dans ce mouvement en développant un prototype de version « sémantique » du portail numeriques.be.

\section{En guise de conclusion}

La numérisation des patrimoines culturels offre des opportunités sans pareil pour leur exploitation et leur valorisation. Des chercheurs peuvent ainsi étudier des objets auxquels l'accès leur était interdit auparavant simplement pour des raisons de préservation physique (pensons ainsi à la numérisation des manuscrits de Qumrân); le public peut admirer des œuvres exposées dans des musées situés à l'autre bout du monde... De nouveaux modes de consommation émergent aussi, que ce soit à la maison devant son PC ou avec des tablettes ou des smartphones lors de visites dans des institutions culturelles.

Dès lors, à quoi ressemblera demain l'accès à la culture numérique ? Rappelons-nous à ce sujet ce que le physicien danois Niels Bohr disait en forme de boutade : «La prévision est un art bien difficile, surtout quand elle concerne l'avenir ", et gageons que les nouveaux usages seront vraisemblablement ceux auxquels nous n'aurions jamais pensé aujourd'hui.

\section{BIBLIOGRAPHIE}

ENUMERATE 2015 : Survey Report on digitisation in European cultural heritage institutions, 2015, téléchargeable à l'adresse http://enumeratedataplatform.digibis.com/reports 
OAIS 2012 : Système ouvert d'archivage de l'information (SOAI) - modèle de référence ; norme ISO 14721, 2012, téléchargeable moyennant paiement à l'adresse www.iso.org/iso/fr/home/ store/catalogue_ics/catalogue_detail_ics.htm ?csnumber $=57284$. La version préliminaire établie par le Comité consultatif pour les systèmes de données spatiales est toutefois téléchargeable en libre accès à l'adresse http://public.ccsds.org/publications/archive/650x0m2.pdf

PEP's 2007 : Suivi des États généraux de la culture : Préservation et Exploitation des Patrimoines Plan PEP's, octobre 2007, téléchargeable à l'adresse www.peps.cfwb.be/index.php ?id =3111

PEP's 2009 : Normes et lignes directrices techniques et organisationnelles pour les initiatives de numérisation des patrimoines culturels soutenues par la Communauté française, téléchargeable à l'adresse www.peps.cfwb.be/index.php ?id =3360

\section{NOTES}

1. Selon le dernier rapport ENUMERATE 2015 sur la numérisation dans les institutions culturelles en Europe, en moyenne $23 \%$ des collections patrimoniales sont numérisées, et environ $50 \%$ des collections doivent encore l'être (ce n'est pas nécessaire pour les $27 \%$ restants).

2. La norme OAIS (OAIS 2012) est un modèle de référence pour un OAIS (Open Archival Information System - système ouvert d'archivage de l'information), qui a propose un modèle d'organisation de l'information pour un AIP (Archival Information Package - paquet d'information archivée) et décrit les fonctions et responsabilités du système d'archivage. Il s'agit d'une norme ISO.

3. LTO (Linear Tape Open) est une technologie de stockage sur bande magnétique au format ouvert. Une cartouche LTO6 a une capacité native de 2,5 To, et une durée de vie estimée de 30 ans.

4. RDF (Ressouce Description Framework) est un modèle de graphe permettant de décrire toute ressource sur le web sous une forme de type <sujet $><$ prédicat $><$ objet $>$

5. OWL (Ontology Web Language) est un langage permettant de formaliser un domaine de connaissances en des classes et des relations entre ces classes, de définir des individus appartenant à ces classes et affirmer des propriétés les concernant, et de raisonner sur ces classes et individus.

\section{RÉSUMÉS}

La Fédération Wallonie-Bruxelles a mis en place depuis une dizaine d'années le plan PEP's (Préservation et Exploitation des Patrimoines) qui consiste à donner un cadre financier et stratégique à l'action des pouvoir publics en mutualisant les moyens et en organisant la numérisation autour de standards communs: l'un des responsables du projet décrit les différentes étapes de ce plan, ses principaux résultats et les perspectives d'avenir concernant les nouveaux usages offerts notamment par le web sémantique. 


\section{AUTEUR}

\section{JEAN-LOUIS BLANCHART}

directeur de la Délégation générale à la numérisation des patrimoines culturels, ministère de la Fédération Wallonie-Bruxelles

jean-louis.blanchart@cfwb.be 